\title{
ANÁLISE E PERSPECTIVA SOBRE A FORMAÇÃO DO PROFISSIONAL DE SAÚDE PARA O ATENDIMENTO À MULHER NEGRA
}

\author{
Helen Hana Fernandes Tavares \\ Universidade de Rio Verde \\ helenhana.ft@gmail.com \\ Bibiana Arantes Moraes \\ Universidade de Rio Verde \\ bibianamoraes@unirv.edu.br
}

Amanda Gomez Matias
Universidade de Rio Verde
amanda12.gm@gmail.com

Hellen Bárbara Souza Silva Universidade de Rio Verde hellenhbsmile@gmail.com

Lana Nicolle Gomes Bernardo Universidade de Rio Verde lananicollegb@gmail.com

\section{Resumo}

A mulher, principalmente a negra, apresenta-se em uma situação de vulnerabilidade tanto na questão econômica, social, cultural e na área da saúde. Para um atendimento integral desta população, o profissional de saúde precisa ser qualificado, para orientar as mulheres a adotarem comportamentos preventivos e de promoção da saúde. Este trabalho teve o objetivo de descrever a importância da formação do profissional de saúde para proporcionar atendimento qualificado à mulher negra. O menor acesso aos serviços de saúde e com isso a demora no diagnóstico/tratamento de determinadas patologias prevalentes na mulher negra, condicionadas a situação de vulnerabilidade causada por fatores como racismo, gênero e desigualdade de classes necessita, portanto de conscientização da população e dos profissionais de saúde, quanto às diferenças inter-étnicas e seu impacto na saúde, além da formulação e execução de atividades educativas que gerem mudanças e promovam práticas equânimes no Sistema Único Saúde (SUS).

Palavras-chave: Saúde da Mulher Negra. Profissional de Saúde. Racismo. Vulnerabilidade. Educação Permanente.

\section{ANALYSIS AND PERSPECTIVE ON THE TRAINING OF THE HEALTH PROFESSIONAL FOR BLACK WOMAN CARE}

\begin{abstract}
The woman, especially the black woman, is in a situation of vulnerability in the economic, social, cultural and health issues. For a comprehensive care of this population, the health professional needs to be qualified, to guide the women to adopt preventive and health promotion behaviors. This study aimed to describe the importance of training the health professional to provide qualified care to black women. The lower access to health services and the delay in the diagnosis / treatment of certain conditions prevalent in black women, conditioned by the situation of vulnerability caused by factors such as racism, gender and class inequality, needs to raise awareness among the population and professionals inter-ethnic differences and their impact on health, as well as the formulation and execution of educational activities that generate changes and promote equitable practices in the Unified Health System (SUS).
\end{abstract}

Keywords: Black Woman's Health. Health Professional. Racism. Vulnerability. Permanent Education.

\section{ANÁLISIS Y PERSPECTIVA SOBRE LA FORMACIÓN DEL PROFESIONAL DE SALUD PARA LA ATENCIÓN A LA MUJER NEGRA}

\section{Resumen}

La mujer, principalmente la negra, se presenta en una situación de vulnerabilidad tanto en la cuestión económica, social, cultural y en el área de la salud. Para una atención integral de esta población, el profesional de salud necesita ser calificado, para orientar a las mujeres a adoptar comportamientos preventivos y de promoción de la salud. Este trabajo tuvo el objetivo de describir la importancia de la formación del profesional de salud para proporcionar atención calificada a la mujer negra. El menor acceso a los servicios de salud y con ello la demora en el diagnóstico / tratamiento de determinadas patologías prevalentes en la mujer negra, condicionadas a la situación de vulnerabilidad causada por factores como racismo, género y desigualdad de clases necesita, por lo tanto, de concientización de la población y de los profesionales de salud, en cuanto a las diferencias interétnicas y su impacto en la salud, además de la formulación y ejecución de actividades educativas que generen cambios y promuevan prácticas ecuánimes en el Sistema Único Salud (SUS).

Palabras clave: Salud de La Mujer Negra. Profesional de Salud. Racismo. Vulnerabilidad. Educación Permanente. 
Análise e perspectiva sobre a formação do profissional de saúde para o atendimento à mulher negra

\section{INTRODUÇÃO}

O Brasil é um país cuja construção da identidade étnico-racial é bastante complexa, podese considerar que é um país mestiço, biológica e culturalmente. Biologicamente é, inegável, o resultado das trocas genéticas entre diferentes grupos populacionais catalogados como raciais, que na vida social se revelam também nos hábitos e nos costumes (SANTOS, 2012).

As mulheres negras trazem consigo a diferença associada por fatores como a classe social a que pertencem, a desigualdade de gênero e a desigualdade racial. Com tantas mudanças durante a história de vida dessas mulheres, passando da escravidão à busca e conquista de seus direitos, há necessidade de atenção integral a elas, visando o atendimento a questões relevantes no campo da saúde, que ainda não foram providas como a redução dos índices de mortalidade materna e o diagnóstico precoce de doenças prevalentes neste grupo como a hipertensão e a diabete (FRAGA, 2014).

Dados do Ministério da Saúde de 2004 demonstram que a mulher negra recebe menos tempo de atendimento médico, como por exemplo, o acompanhamento no parto, cuja $46,2 \%$ das mulheres brancas tiveram o acompanhamento, enquanto que nas mulheres negras apenas $27 \%$. Com relação a orientações a respeito do aleitamento materno, mostrou que 62,5\% das mulheres negras receberam essa informação, comparado a 77,7\% das mulheres brancas. Com relação a mortes maternas 60\% ocorrem entre mulheres negras e 34\% entre as brancas (BRASIL, 2004).

Assim, justificava-se a importância de se conhecer os determinantes da fragilidade de saúde da mulher negra, bem como a necessidade de maiores estudos e de um olhar singular por parte dos profissionais de saúde. O conhecimento e as informações destes profissionais capacitados a atender a mulher negra irão nortear o raciocínio clínico e o atendimento integralista e orientações. O intuito de serem adotadas atitudes de estímulo ao acesso a serviços de saúde de forma precoce é de contribuir para uma melhor qualidade de vida.

Frente ao exposto, este trabalho teve o objetivo de descrever a importância da formação do profissional de saúde para proporcionar atendimento qualificado as populações vulneráveis, em especial a mulher negra.

\section{CONTEXTUALIZAÇÃO}

A sociedade brasileira é caracterizada por uma pluralidade étnica, por ser resultado de um processo histórico que inseriu num mesmo cenário três povos distintos: portugueses, índios e negros. Os africanos foram dominados em forma de escravidão, sem qualquer respeito a suas 
Análise e perspectiva sobre a formação do profissional de saúde para o atendimento à mulher negra

diferenças culturais e foram identificados como negros, crioulos ou pretos. Desde o princípio da formação histórica brasileira estabelece-se uma associação entre a posição do indivíduo e a cor da pele, por meio de várias gerações, foram identificados e tratados de forma sempre negativa em todos os planos (CONCEIÇÃO, 2010).

A identidade negra é uma construção social, histórica e cultural, em que o sujeito se reconhece na conjunção do grupo étnico/racial a partir da sua história, cultura e relações estabelecidas com o outro (MUNANGA, 2012). Assim, afirmar a identidade negra faz parte de um processo de ruptura com os estigmas históricos dessa população que foi e é inferiorizada e subjugada diante de um ideal estético-cultural eurocêntrico, desde o Brasil colonial (SANTOS, 2012).

Entretanto, apesar da definição de quem é negro ser teoricamente de fácil entendimento, o auto-reconhecimento como tal, torna-se difícil em um país que desenvolveu o desejo de branqueamento. O branqueamento, é quando o negro descontente e desconfortável com sua condição racial, não se reconhece como negro e até procura miscigenar-se com pessoas brancas. Associado a isso, há no imaginário popular pré-conceitos extremamente negativos sobre o negro que é propagado e interferem nas relações raciais. Dessa forma, tanto a dimensão subjetiva quanto a objetiva do branqueamento, reforçam mutuamente para atuarem como potencializadoras da reprodução do racismo e das desigualdades raciais no Brasil (BENTO, 2014).

Os negros são os que ocupam as posições menos qualificadas e pior remuneradas no mercado de trabalho (mulheres recebem 60\% menos e homens 15\%); os que apresentam níveis mais baixos de instrução (média de 4,7 anos de estudo em contraposição aos 6,9 anos dos brancos); que residem em áreas com menos serviços de infraestrutura básica; que sofrem maiores restrições ao acesso à serviços de saúde e, quando o recebem, são de pior qualidade. Além disso, é a parcela da população que possui maiores taxas de mortalidade evitáveis como: doenças cardiovasculares, causas externas, mortalidade infantil e materna, HIV/ AIDS (entre as mulheres), tuberculose, doença cerebrovascular e diabetes (BRASIL, 2005; MEDEIROS, 2015).

No Brasil, as diferenças inter-raciais advêm de um histórico de hierarquização social fundamentado no racismo, como fenômeno ideológico que interliga raça, classe social e gênero e que obtêm como único resultado a multiplicação de vulnerabilidades. A "naturalidade" deste tipo de hierarquia social determina que a parcela da população mais afetada, a da mulher negra, logo, como consequência, ela se encontra prejudicada no conjunto de aspectos individuais e coletivos relacionados ao grau e modo de exposição a uma dada situação e, de modo inseparável, ao acesso 
Análise e perspectiva sobre a formação do profissional de saúde para o atendimento à mulher negra

a recursos adequados para se proteger das consequências indesejáveis daquela situação (LOPES, 2004).

Ao avaliar a vulnerabilidade e consequente o adoecimento do qual as mulheres negras estão submetidas, utiliza-se três planos interdependentes de análise: o componente individual, o componente social ou coletivo e o componente político ou programático. No âmbito individual trata-se da população que está desprotegida, exposta ao risco de discriminação ou de tratamento injusto que desafie os princípios de igualdade e dignidade humana. Em termos sociais, são aqueles que não têm seus direitos efetivados e nem acesso aos serviços e bens sociais disponíveis (PRESTES, 2016; BRASIL, 2005).

O componente político ou programático trata-se da falta de investimentos em ações e programas de informação e educação preventivas; ausência de serviços sociais e de saúde de fácil acesso, com alta qualidade, democratizados, periodicamente retroalimentados e avaliados; e falha na adoção de mecanismos de não discriminação nas instituições. Em decorrência de todos esses componentes é que no Brasil considera-se discriminação como um fator provocador de fragilidade da saúde, tornando assim as mulheres negras a parcela da população que necessita de maior grau de proteção e assistência à saúde (PRESTES, 2016; BRASIL, 2005).

O Sistema Único de Saúde (SUS) juntamente com o Ministério da Saúde ao longo dos anos e de sucessivos movimentos sociais de mulheres em geral, criaram mecanismos de intervenção à saúde desta população como o Programa de Assistência Integral à Saúde da Mulher (PAISM) criado em 1984, que ampliando em 2004 tornou-se a Política Nacional de Assistência Integral à Saúde da Mulher (PNAISM). Também foi criada especificamente a Atenção à Saúde das Mulheres Negras em 2005 e a Política Nacional de Saúde Integral da População Negra (PNSIPN) em 2009.

\footnotetext{
A Política Nacional de Saúde Integral da População Negra define os princípios, a marca, os objetivos, as diretrizes, as estratégias e as responsabilidades de gestão voltada para a melhoria das condições de saúde desse segmento da população. Inclui ações de cuidado, atenção, promoção à saúde e prevenção de doenças, bem como de gestão participativa, participação popular e controle social, produção de conhecimento, formação e educação permanente para trabalhadores de saúde, visando à promoção da equidade em saúde da população negra (BRASIL, 2007).
}

A saúde vai além de um fator biológico-natural, logo surge considerar que ela faz parte da coexistência do indivíduo em sociedade e ao acesso às redes socioeconômicas e de serviços essenciais. Portanto, o contexto social e a história de vida individual e coletiva irão influenciar positiva ou negativamente na saúde. Logo, há uma produção social da enfermidade, ou da manutenção do bem-estar, nas sociedades de classes, da opressão racial/ étnica e da opressão de gênero. (ROCHA; DAVID, 2015). 
Análise e perspectiva sobre a formação do profissional de saúde para o atendimento à mulher negra

A garantia à saúde de forma universal e equitativo está diretamente associada ao acesso e utilização dos serviços que, muitas vezes, são infringidos por vários fatores que se articulam como racismo, sexismo, condições sócio-econômicas e culturais. A utilização dos serviços e insumos de saúde são condições importantes para a manutenção de bom estado de saúde ou para seu reestabelecimento, embora não seja o único fator responsável por uma vida saudável e de boa qualidade (GOES, 2013).

A população negra, quando comparada à população branca, apresenta indicadores sociais piores. As mulheres negras são vítimas recorrentes do racismo e do sexismo na sociedade, o que leva à dificuldade no acesso aos serviços de saúde do SUS (BRASIL, 2016). A Política Nacional de Atenção Integral à Saúde da Mulher considera a diversidade de mulheres na sociedade brasileira e suas diferentes condições de saúde, além da importância racial para análise dos indicadores e consequentemente avaliação da situação de saúde de grupos populacionais.

Quando comparadas às mulheres brancas, a maioria das mulheres negras encontra-se mais abaixo da linha de pobreza e a taxa de analfabetismo é dobrada. Em razão disto, elas possuem menor acesso aos serviços de saúde de boa qualidade (BRASIL, 2004). A medicina baseada em evidências demonstra que algumas doenças são mais frequentes, ou evoluem de forma diferenciada nas mulheres negras por fazerem parte do agrupamento humano racial negro e/ ou serem do sexo feminino. Isto ocorre devido à existência de determinadas interações ambientais e culturais com o patrimônio genético, sendo que as de maior prevalência são hipertensão arterial, diabetes mellitus tipo II, anemia falciforme e morte materna. (LIMA, 2014).

Estudos revelam que a taxa de mortalidade por síndromes hipertensivas nas negras é quase seis vezes maior do que nas brancas devido à um início precoce e uma evolução de maior gravidade (BRASIL, 2004).

\footnotetext{
A etnia negra é um forte fator predisponente à hipertensão arterial, deixando as pessoas afro-brasileiras expostas ao desenvolvimento de uma hipertensão mais severa, como também a um maior risco de ataque cardíaco e morte súbita quando comparadas às pessoas de etnia branca (PITHAN, et al; 2014).
}

As negras têm $50 \%$ a mais de chances de desenvolver diabetes que as brancas. Autores estimam uma maior proporção de obesos nesta população e uma menor frequência de diagnósticos prévios e tratamentos específicos, devido às diferenças socioeconômicas estabelecidas entre negros e brancos, refletida nas diferenças ao acesso nos serviços de saúde (PITHAN, et al; 2014).

A anemia falciforme incide majoritariamente em negros em todo o mundo e apresenta alto índice de mortalidade. Os especialistas alertam que a melhor estratégia para atender quem 
Análise e perspectiva sobre a formação do profissional de saúde para o atendimento à mulher negra

tem anemia falciforme é o diagnóstico e o cuidado precoce, o que muitas vezes falta a esta população (PITHAN, et al; 2014).

A taxa de mortalidade materna entre as mulheres negras pode ser até seis vezes maior do que entre as brancas. Dentre os fatores para a ocorrência dessa mortalidade pode-se citar: hipertensão, diabetes, doenças sexualmente transmissíveis, dificuldades econômicas, nível educacional, cardiopatias associadas, obesidade, síndromes hemorrágicas relacionadas ao parto, infecções no puerpério e complicações decorrentes de abortos realizados incorretamente. Uma boa orientação e uma adequada avaliação pré-concepcional para todas as mulheres e principalmente para as negras, poderá diminuir essas taxas (PITHAN, et al; 2014).

A eficácia do cuidado em saúde varia de acordo com as condições socioeconômicas do sujeito, o lugar onde ele vive, a qualidade dos equipamentos sociais que ele tem acesso, a sensibilidade, a humanização e o compromisso dos profissionais pelos quais ele é atendido. A análise da estrutura social e sua evolução no tempo são imprescindíveis (BRASIL, 2005).

Não se deve afastar os sujeitos uns dos outros, do seu ambiente. O registro e a análise das desigualdades e iniquidades raciais em saúde só pode ser feitos por pessoas sensíveis e aptas que compreendem os variados sentidos e significados atribuídos às relações inter e intra-raciais, inter e intragêneros, inter e intrageracionais (LOPES; MALACHIAS, 2001).

O preconceito por vezes até inconsciente, a incompreensão, a falta de atenção ou a aceitação de estereótipos racistas que colocam uma parcela da população em desvantagem; a subrepresentação de negros entre os cuidadores de nível superior e a falta de aptidão para o manejo de questões relacionadas à raça ou etnia testificam para a elaboração de estratégias menos evidentes. No caso da saúde, o uso da linguagem como meio de continuidade e comprovação das desigualdades sociais e raciais permite que sejam criados, quotidianamente, novos instrumentos para o não oferecimento de um serviço profissional, adequado e igualitário (BRASIL, 2005). Para tentar resolver estas questões é necessário:

1 - Adequar a oferta de serviços de forma a garantir o acesso de populações excluídas do Sistema, como a população negra, observando o cuidado em todos os graus de complexidade das necessidades;

2 - Favorecer a intersetorialidade no planejamento de ações de modo a melhorar a eficiência e eficácia dos serviços oferecidos às populações vulnerabilizadas, sobretudo à população negra;

3 - Instruir profissionais para melhoria da qualidade de informação, enfatizando a importância do preenchimento da variável cor, entre outras; 
Análise e perspectiva sobre a formação do profissional de saúde para o atendimento à mulher negra

4 - Certificar que os Pólos de Educação Permanente qualifiquem trabalhadores e gestores do SUS com o intuito de eliminar práticas discriminatórias que se reproduzem na rede de saúde (BRASIL, 2005).

A versão do PNS (Plano Nacional de Saúde) discutida durante o ano de 2004 insiste em dizer da necessidade de iniciativas de longo, médio e curto prazos, além da adoção de ações afirmativas em saúde, a fim de atendimento de demandas mais imediatas. É essencial a formação permanente e contínua dos profissionais de saúde do SUS para que possam prestar o atendimento adequado considerando sempre as especificidades genéticas, derivadas de exposição ocupacional a fatores degradantes, aquelas derivadas da indisponibilidade de recursos socioeconômicos, da desnutrição, da pressão social (BRASIL, 2005).

Destaque-se também a necessidade de assegurar a efetiva participação da população negra no planejamento das ações e no seu monitoramento. O PNS orienta gestores, gerentes e outros profissionais de saúde para a inserção do quesito raça/cor nos instrumentos de coleta de informação e na análise dos dados em saúde, com o intuito de facilitar a realização de pesquisas que subsidiem o aperfeiçoamento da promoção e da atenção à saúde (BRASIL, 2005; NOGUEIRA, 2004).

Quando se analisa a mulher negra de uma forma mais aprofundada, descobre-se que os maiores fatores responsáveis pela prevalência de determinadas patologias se dá pela situação de vulnerabilidade causada por fatores como racismo, pela questão de gênero que o sexo feminino enfrenta e a desigualdade de classes existente na sociedade atual. Para o enfrentamento destas questões no campo da promoção da saúde demanda-se inovações, alargamento de perspectivas, reformulações de teorias, técnicas e práticas (FRAGA, 2014; PRESTES, 2016).

Um outro fator além do preconceito, exclusão e pobreza provocados pela desigualdade existente pelo racismo e segregação de classes são os fatores sistêmicos no que tange o sucateamento e a incapacidade do sistema de saúde enfrentar a realidade brasileira nas condições vivenciadas pelo cidadão brasileiro. (LIMA, 2014).

Torna-se necessário a produção de conhecimento, o desenvolvimento de metodologias com conteúdos sobre diferenças inter-étnicas nas condições de vida e na saúde da população, ações especialmente orientadas a corrigir as desigualdades que se verificam na condição de mulheres e negros e principalmente, a capacitação de profissionais da rede básica e dos serviços de referência e das maternidades, sensibilizando-os para introduzir o quesito cor e realizar diagnósticos com recorte racial e equidade (BRASIL, 2005; NOGUEIRA, 2004).

A Educação Permanente em saúde com inclusão dos temas Racismo e Saúde da População Negra nos conteúdos e processos de aprendizagem, bem como o esforço em 
Análise e perspectiva sobre a formação do profissional de saúde para o atendimento à mulher negra

compreender e apaziguar os processos históricos de censura ou de discriminação institucionais baseados na cor da pele são aspectos chaves na contribuição para a formulação e execução de atividades educativas que gerem mudanças e promovam práticas equânimes no SUS, além de contribuir para uma melhor interação profissional-paciente. É ínfimo pensar apenas na prescrição de medicamentos, reeducação, treinamento de comportamento preventivo e deixar de analisar o impacto das relações raciais, de gênero no processo saúde-doença (FRAGA, 2014).

É necessário que ocorra a conscientização da população e dos profissionais de saúde, quanto às diferenças inter-étnicas. Um exemplo seria a promoção de palestras ou outros eventos educativos esclarecendo as doenças que a raça negra está mais propensa a adquirir ao longo da vida. Nessas palestras haveria também a promoção e prevenção da saúde resultando, por parte dos profissionais, um melhor manejo da atenção à mulher negra. Além disso, as palestras levariam a parcela da população vulnerável a se proteger mais, de acordo com as suas peculiaridades raciais (PITHAN, et al; 2014; FRAGA, 2014).

A capacitação dos profissionais de saúde para cuidar de mulheres negras se faz de extrema importância. É vital que estes estejam atentos aos riscos maiores de adquirir certas enfermidades nessas pessoas, seja na saúde do dia-a-dia ou na gestação e puerpério. É claro que os hábitos de vida também influenciam nessa questão. Contudo, se os cuidadores da saúde souberem da propensão que determinadas raças têm à algumas doenças, eles poderão implementar uma atenção primária à saúde bem mais eficiente com tais indivíduos. Isso com certeza teria um grande impacto na saúde comunitária evitando assim diversos agravos de doenças prevalentes em pessoas negras do sexo feminino (FRAGA, 2014).

\section{CONSIDERAÇÕES FINAIS}

O racismo pode ser compreendido como um determinante social e pode contribuir no processo saúde-doença ao se observar a saúde das mulheres negras sob o prisma étnico-racial. As formas como as relações raciais estão ancoradas no Brasil tornam a população negra mais vulnerável e tendem a incidir sobre acesso e qualidade nos serviços de saúde.

Para garantir um atendimento ético, humanizado e de qualidade à este grupo de mulheres são necessárias iniciativas por parte do Ministério da Saúde no intuito de formar, capacitar e orientar profissionais de saúde a executar a Política Nacional de Saúde Integral da População Negra, com cursos voltados para gestores, profissionais e sociedade civil, abrangendo temas de interesse no diagnóstico e tratamento das doenças prevalentes, promoção da saúde, com foco nos condicionantes e determinantes de saúde, com vistas no atendimento humanizado e qualificado 
Análise e perspectiva sobre a formação do profissional de saúde para o atendimento à mulher negra

pelos profissionais de saúde na atenção básica. Além de reforçar o trabalho na promoção da equidade e enfrentamento à discriminação nas instituições e serviços do SUS.

Portanto, é essencial a formação permanente e contínua dos profissionais de saúde para que possam prestar o atendimento adequado considerando sempre as especificidades genéticas e as indisponibilidades de recursos socioeconômicos. Além do atendimento, esta formação aos profissionais de saúde será de relevância para o acesso e o atendimento com vistas a diminuir vulnerabilidades da população negra. Este trabalho só levanta alguns pontos da vulnerabilidade da mulher negra no quesito saúde, outros estudos ainda precisam ser levantados para que essa população seja melhor cuidada.

\section{REFERÊNCIAS}

BENTO, Maria Aparecida Silva. Branqueamento e branquitude no Brasil. Caderno de textos Racismo Institucional. Disponível em: <http://www.cehmob.org.br/wpcontent/uploads/2014/08/Caderno-Racismo.pdf\#page=5> . Acesso: 17 de março de 2017.

BRASIL. Fundação Nacional de Saúde. Saúde da população negra no Brasil: contribuições para a promoção da equidade / Fundação Nacional de Saúde. - Brasília: Funasa, 2005.

BRASIL. Ministério da Saúde. Secretaria de Atenção à Saúde. Departamento de Ações Programáticas Estratégicas. Política nacional de atenção integral à saúde da mulher: princípios e diretrizes. Brasília: Ministério da Saúde, 2004.

BRASIL. Ministério da Saúde. Secretaria de Gestão Estratégica e Participativa. Departamento de Articulação Interfederativa. Temático Saúde da População Negra. - Brasília: Ministério da Saúde, 2016. 82 p.: il. (Painel de Indicadores do SUS; v. 7, n. 10) ISBN 978-85-334-2359-6.

BRASIL. Ministério da Saúde. Secretaria de Gestão Estratégica e Participativa. Política Nacional de Saúde Integral da População Negra. Brasília: Ministério da Saúde, 2007.

BRASIL. Ministério da Saúde. Ação do Ministério da Saúde e Secretaria de Direitos Humanos tem início no mês da Consciência Negra e marca os dez anos da Política de Saúde da População Negra. Portal da saúde. Brasília: Ministério da Saúde, 2004.

CONCEIÇÃO, Helenice da Cruz; CONCEIÇÃO, Antônio Carlos Lima. A construção da identidade afrodescendente. Revista África e Africanidades - Ano 2 - n. 8, fev. 2010 - ISSN 1983-2354.

FRAGA, Fabiana Albino; SANINO, Giane Elis de Carvalho. Saúde da mulher negra passos e descompassos: ações afirmativas na saúde, provável luz no fim do túnel? Revista da ABPN • v. 7, n. $15 \cdot$ nov. 2014 - fev. 2015, p.192-211.

GOES, Emanuelle Freitas; NASCIMENTO, Enilda Rosendo. Mulheres negras e brancas e os níveis de acesso aos serviços preventivos de saúde: uma análise sobre as desigualdades. Saúde 
Análise e perspectiva sobre a formação do profissional de saúde para o atendimento à mulher negra

debate vol.37 no.99 Rio de Janeiro Oct./Dec. 2013. http://dx.doi.org/10.1590/S010311042013000400004 .

LIMA, Adriana Silva Guedes; VOLPATO, Luci Martins Barbatto. Saúde da mulher Negra e os determinantes: Racismo, Questão de Gênero e Classe Econômica. ETIC Vol. 10, No 10 (2014): ETIC - Encontro de Iniciação Científica - ISSN 21-76-8498.

LOPES, Fernanda. Experiências desiguais ao nascer, viver, adoecer e morrer: tópicos em saúde da população negra no Brasil. Caderno de Textos Básicos do I Seminário Nacional de Saúde da População Negra. Brasília- DF, 2004.

LOPES, F; MALACHIAS, R. Assumir a diferença para promover a igualdade: a importância do quesito cor na investigação epidemiológica. Boletim Epidemiológico de AIDS do PEDST/AIDS. Secretaria do Estado da Saúde. São Paulo 2001 out; Ano XIX(2): 3-5.

MEDEIROS, Camila Pinheiro. Mulheres Negras: racismo, identidade e discurso étnico I. Disponível em: http://cienciassociais.ufsc.br/files/2015/03/Artigo-19.pdf. Acesso em: 11 abril 2017.

MUNANGA, kabengele. Negritude e Identidade Negra ou Afrodescendente: um racismo ao avesso? Revista da ABPN • v. 4, n. 8 • jul.-out. 2012 • p. 06-14.

NOGUEIRA, João Carlos. Movimento negro: das denúncias do racismo à prática de políticas públicas. Capa $>$ v. 3, n. 5 (2004) > Nogueira. Disponível em: https://periodicos.ufsc.br/index.php/politica/article/view/1980. Acesso em: 11 abril 2017.

PITHAN, Circe Ottonelli; SOARES, Elaine Oliveira; URATANI, Fernanda Niederauer; FRANCO, Luciane Rampanelli ; AMARAL, Nilton Vieira; VILARINO, Rosa Maria Rimolo; Diretrizes para acolhimento e assistência à saúde das mulheres negras, Prefeitura Municipal de Porto Alegre, Secretaria Municipal de Saúde, 2014.

PRESTES, Clélia R.S.; PAIVA, Vera S.F. Abordagem psicossocial e saúde de mulheres negras: vulnerabilidades, direitos e resiliência. Saúde Soc. São Paulo, v.25, n.3, p.673-688, 2016.

ROCHA, Patrícia Rodrigues; DAVID, Helena Maria Scherlowski Leal. Determinação ou Determinantes? Uma discussão com base na Teoria da Produção Social da Saúde. Rev Esc Enferm USP · 2015; 49(1):129-135.

SANTOS, Silvia Karla B. M. M. O que é ser negro no Brasil? - Uma reflexão sobre o processo de construção da identidade do povo brasileiro. Cadernos Imbondeiro. João Pessoa, v.2, n.1, 2012. 1. 